Research Article

\title{
Computational Approach in Formulating Mechanical Characteristics of 3D Star Honeycomb Auxetic Structure
}

\author{
Mozafar Shokri Rad, Zaini Ahmad, and Amran Alias \\ Faculty of Mechanical Engineering, Universiti Teknologi Malaysia, 81310 Johor Bahru, Malaysia \\ Correspondence should be addressed to Zaini Ahmad; azaini@fkm.utm.my
}

Received 9 September 2014; Revised 4 January 2015; Accepted 5 January 2015

Academic Editor: Rui Vilar

Copyright ( 2015 Mozafar Shokri Rad et al. This is an open access article distributed under the Creative Commons Attribution License, which permits unrestricted use, distribution, and reproduction in any medium, provided the original work is properly cited.

\begin{abstract}
Auxetic materials exhibit a unique characteristic due to the altered microstructure. Different structures have been used to model these materials. This paper treats a development of finite element model and theoretical formulation of 3D star honeycomb structure of these materials. Various shape parameters of the structural cell were evaluated with respect to the basic mechanical properties of the cell. Finite element and analytical approach for various geometrical parameters were numerically used to formulate the characteristics of the material. The study aims at quantifying mechanical properties for any domain in which auxetic material is of interest for variations in geometrical parameters. It is evident that mechanical properties of the material could be controlled by changing the base wall angle of the configuration. The primary outcome of the study is a design guideline for the use of 3D star honeycomb auxetic cellular structure in structural applications.
\end{abstract}

\section{Introduction}

For decades, several geometrical structures representing auxetic behaviour have been introduced, fabricated, and tested particularly to obtain their mechanical properties. These geometrical structures are beneficial since they make contribution to researchers to comprehend on how auxetic behaviour can be obtained and how auxetic materials can be fabricated. Computational procedure is the main contribution in predicting their properties.

Reentrant structures which have been investigated by various researchers [1-6], chiral structures [7-9], rotating $\mathrm{rigid} / \mathrm{semi}$-rigid units [10-14], angle-ply laminates [15, 16], hard molecules [17-20], microporous polymers [21-23], and liquid crystalline polymer [24-26] are among the most important classes of such auxetic structures. Star honeycomb constituent of reentrant structural group has increasingly received attention compared to other structures due to its exceptional ability to model auxetic materials. Within the same group, other structures which are different from star honeycomb structures are double arrow head structures, structurally hexagonal honeycomb, structures formed from lozenge grids, structure formed from square grids, and structure formed from sinusoidal ligaments [27]. Man-made auxetic materials were first designed by Theocaris et al. in 1997 [28]. In their study, the design of material was based on structure of arrays with polygonal-shaped inclusions and reentrant corners. Based on their research, the moduli of the inclusion material should be lower than the moduli of the material of structure in order to make composite with desired negative Poisson's ratio. Using numerical analysis, they proved that the shape of the star-shaped microinclusions is the main factor causing auxetic behaviour.

In the present study, a simple 3D star honeycomb cellular structure was modelled by adapting a $2 \mathrm{D}$ cellular structure. Subsequently, the influence of structure shape on basic properties has also been evaluated. In particular, the basic mechanical properties discussed in this study are elastic modulus, density ratio, and Poisson's ratio which were examined for different values of shape parameters, namely, the wall length, the cell wall thicknesses, and the cellular structure angle. Consisting of eight elastic beams, the 2D cellular structure has a symmetrical configuration. Analytical formulation of mechanical properties for the structure has eventually been developed. This investigation highlights finite element discretization and the developed formulation of $3 \mathrm{D}$ 

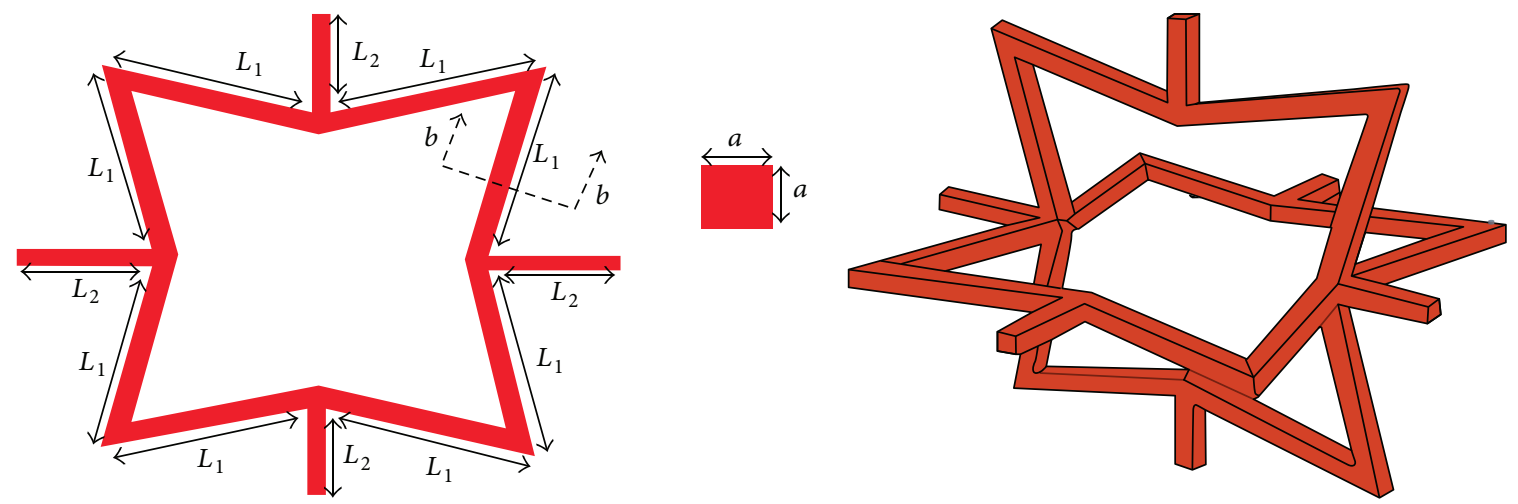

(a)

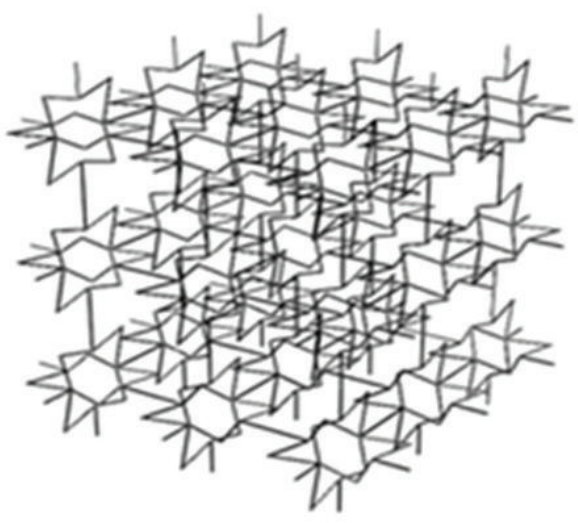

(b)

Figure 1: (a) Star honeycomb structure in 2D and 3D. (b) An element of auxetic material made of 3D star honeycomb structures.

star honeycomb structures for auxetic materials that have not been proposed previously. From this study, it is evident that mechanical properties of auxetic material are controllable, thus facilitating the fabrication technique used in preparing samples in the laboratory.

\section{Definition of the Auxetic Model}

Figure 1 shows beam-like 2D and 3D star honeycomb structures formed by eight (8) elastic beams with the same length and cross-sectional area. By assembling such single unit of structural cell, an element of auxetic material made of $3 \mathrm{D}$ star honeycomb structure is produced as depicted in Figure 1(b). This structural configuration is deemed as cellular structure of a material. From the mechanics point of view, mechanical properties of the cellular material depend on both geometrical parameters of the cells and mechanical properties of the base material [3]. This study has attempted to formulate some of the important mechanical properties of the auxetic material. These parameters include Poisson's ratio, elastic modulus, and density ratio. Based on symmetrical properties of the $3 \mathrm{D}$ cellular structures, it can be assumed that uniaxial loading of the cell along $x$ direction leads to the same deflection along both $y$ and $z$ directions. Also, uniaxial loading along $y$ and $z$ directions results in the same deflections in the lateral directions. As a result, the following set of equations can be written:

$$
\begin{aligned}
& v_{x y} \neq v_{y x}=v_{z x} \\
& v_{y z}=v_{z y} \\
& E_{2}=E_{3} \neq E_{1} .
\end{aligned}
$$

The following equation should be satisfied to obtain a starshaped structure as shown in Figure 1:

$$
L_{1} \sin \theta>L_{1} \cos \theta \Longrightarrow \theta>\frac{\pi}{4} .
$$

\section{Finite Element Modelling}

An elastic cellular structure has numerically been developed using finite element technique to determine the basic properties of the star honeycomb structures. ABAQUS finite element code has been used to perform these analyses. An element of the auxetic material shown in Figure 1 was taken into consideration. The element consists of 27 cellular structures. Element used is the linear tetrahedral, type C3D4. A total number of elements and nodes are 2,916,604 and 746,044 , respectively. The mesh size was determined from the convergence study. It is evident that finite element analysis 


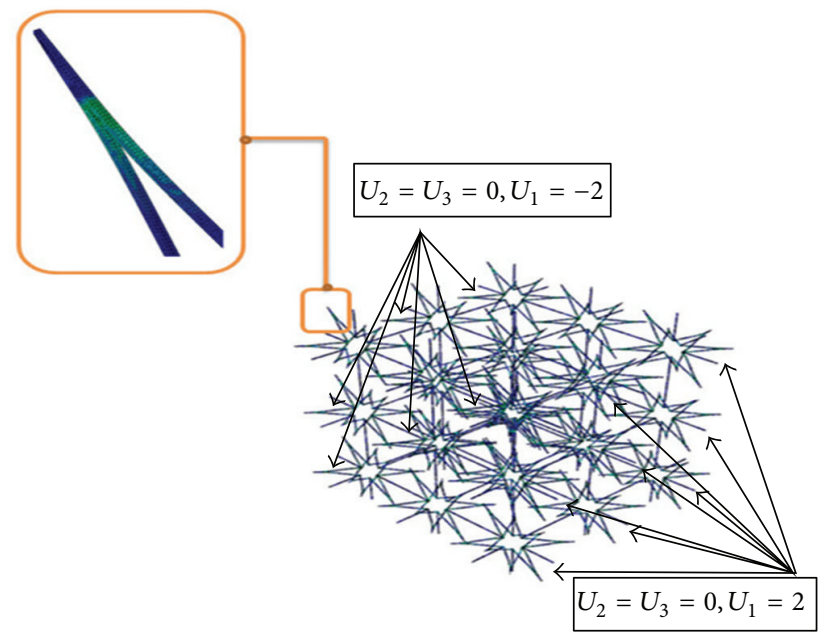

Figure 2: Von Misses stress of Model number 1 in the case of uniaxial imposed displacement along $x$ direction.

of this element leads to more accurate and reliable results compared to the analysis of single cellular structure. Furthermore, the element was loaded along $x$ with the imposed normal deflections. The resulting stresses as well as normal and transverse strains could then be obtained.

Meanwhile, the Young's modulus was calculated by dividing the averaged uniaxial stresses by the uniaxial strains while the Poisson's ratios were obtained by dividing the averaged transverse strain by the uniaxial imposed ones. Several finite element models were consecutively developed for different values of the length, cross section, and angles. The Poisson's ratios and elastic modulus including $v_{x y}, v_{x z}$, and $E_{1}$ were computed using the same method. Figure 2 shows finite element modelling of Model number 1. All elements were then loaded with imposed normal deflections in $y$ direction. The Poisson's ratios and elastic modulus including $v_{y x}, v_{y z}$, and $E_{2}$ were computed using the same method. Figure 3 shows finite element modelling of the same model for loading along $y$ direction. Due to the symmetry of the element, loading along $y$ and $z$ direction provides the same results. As such, the loading along $z$ direction has not been performed in the present study.

\section{Development of Analytical Formulation}

Using analytical approach, Poisson's ratios, elastic modulus, and density ratios of all models may also be predicted. From mathematics and mechanics point of views, there is relationship between the geometrical parameters of the cell, mechanical properties of the base material, and mechanical properties of the star honeycomb structure including Poisson's ratio, elastic modulus, and density ratio. The relationships can be expressed as the following functions:

$$
\begin{aligned}
& v_{x y}=f_{1}\left(L_{1}, L_{2}, a, \theta\right), \\
& v_{x z}=f_{2}\left(L_{1}, L_{2}, a, \theta\right), \\
& v_{y x}=f_{3}\left(L_{1}, L_{2}, a, \theta\right),
\end{aligned}
$$

$$
\begin{aligned}
v_{y z} & =v_{z y}=f_{4}\left(L_{1}, L_{2}, a, \theta\right), \\
E_{1} & =f_{5}\left(E, L_{1}, L_{2}, a, \theta\right), \\
E_{2} & =E_{3}=f_{6}\left(E, L_{1}, L_{2}, a, \theta\right), \\
\rho & =f_{7}\left(\rho_{c}, a, L_{1}, L_{2}\right),
\end{aligned}
$$

where $L_{1}, L_{2}, a$, and $\theta$ are geometrical parameters of the cell shown in Figure 1. $E$ and $\rho_{c}$ are elastic modulus and density of the base material, respectively. $v_{x y}, v_{x z}, v_{y x}, v_{y z}, v_{z x}, v_{z y}$, $E_{1}, E_{2}$, and $E_{3}$ are Poisson's ratios and elastic modulus of the material. Using dimensionless parameters, the functions of Poisson's ratios and elastic modulus of the material could be predicted as follows:

$$
\begin{aligned}
& v_{x y}=F_{1}(\alpha, \beta, \gamma)=\sum_{i=0}^{2} \sum_{j=0}^{2} \sum_{k=0}^{2} A_{i j k}\left[\alpha^{i} \beta^{j} \gamma^{k}\right], \\
& v_{x z}=F_{2}(\alpha, \beta, \gamma)=\sum_{i=0}^{2} \sum_{j=0}^{2} \sum_{k=0}^{2} B_{i j k}\left[\alpha^{i} \beta^{j} \gamma^{k}\right], \\
& v_{y x}=F_{3}(\alpha, \beta, \gamma)=\sum_{i=0}^{2} \sum_{j=0}^{2} \sum_{k=0}^{2} C_{i j k}\left[\alpha^{i} \beta^{j} \gamma^{k}\right], \\
& v_{y z}=F_{4}(\alpha, \beta, \gamma)=\sum_{i=0}^{2} \sum_{j=0}^{2} \sum_{k=0}^{2} D_{i j k}\left[\alpha^{i} \beta^{j} \gamma^{k}\right], \\
& \frac{E_{1}}{E}=F_{5}(\alpha, \beta, \gamma)=\sum_{i=0}^{2} \sum_{j=0}^{2} \sum_{k=0}^{2} E_{i j k}\left[\alpha^{i} \beta^{j} \gamma^{k}\right], \\
& \frac{E_{2}}{E}=F_{6}(\alpha, \beta, \gamma)=\sum_{i=0}^{2} \sum_{j=0}^{2} \sum_{k=0}^{2} F_{i j k}\left[\alpha^{i} \beta^{j} \gamma^{k}\right], \\
& \frac{\rho}{\rho_{c}}=F_{7}(\alpha, \beta, \gamma)=\sum_{i=0}^{2} \sum_{j=0}^{2} \sum_{k=0}^{2} G_{i j k}\left[\alpha^{i} \beta^{j} \gamma^{k}\right],
\end{aligned}
$$

where $\alpha=\theta, \beta=L_{2} / L_{1}$, and $\gamma=a / L_{1}$. 


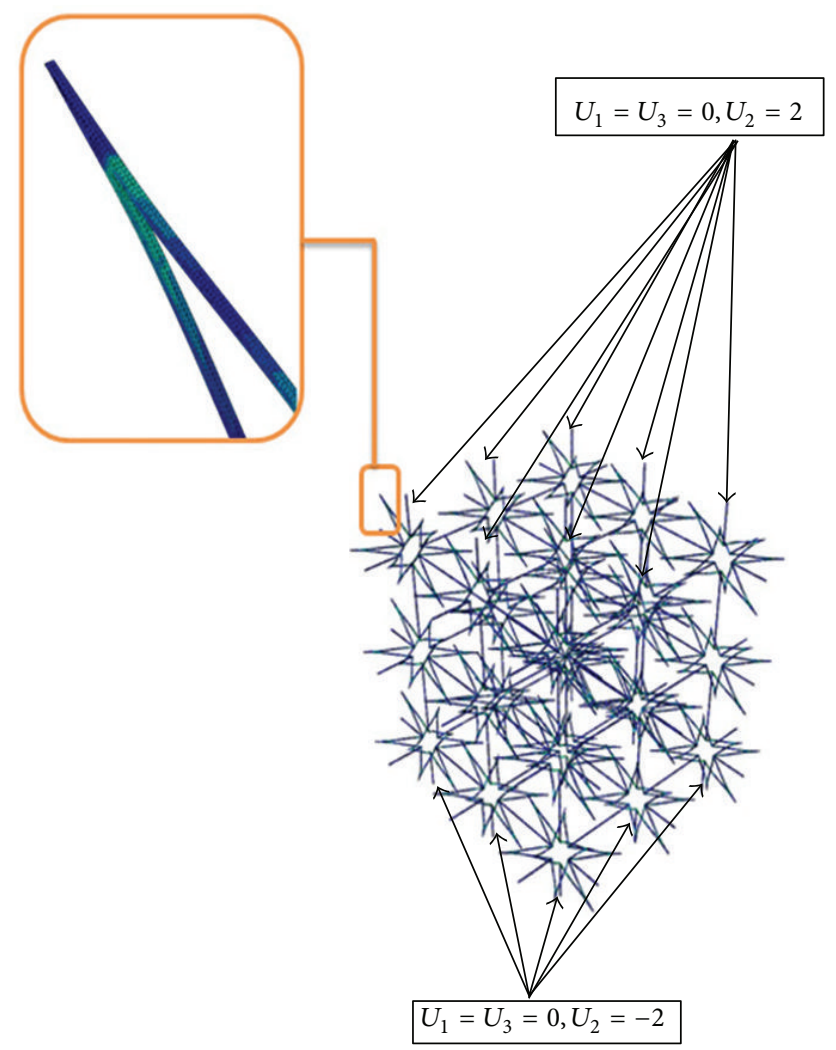

Figure 3: Von Misses stress of Model number 1 in the case of uniaxial imposed displacement along $y$ direction.

For the validity of a set of (4), each function must have twenty-seven (27) coefficients. To obtain these coefficients, values of Poisson's ratios, elastic modulus, and density ratio for twenty-seven (27) models with different geometrical parameters are required. Finite element analysis has been carried out for the models. Table 1 shows the values of $\alpha, \beta$, and $\gamma$ used for inclusion into finite element analysis.

By using a set of (4) for all models, the matrix form of $v_{x y}$ function is written as follows:

$$
\begin{aligned}
& {\left[\begin{array}{cccc}
1 & \gamma_{1} \gamma_{1}^{2} \beta_{1} & \cdots & \left(\left(\alpha_{1}\right)^{2}\left(\beta_{1}\right)^{2}\left(\gamma_{1}\right)^{2}\right) \\
1 & \gamma_{2} \gamma_{2}^{2} \beta_{2} & \cdots & \left(\left(\alpha_{2}\right)^{2}\left(\beta_{2}\right)^{2}\left(\gamma_{2}\right)^{2}\right) \\
\vdots & \vdots & \vdots & \vdots \\
\vdots & \vdots & \vdots & \vdots \\
1 & \gamma_{27} \gamma_{27}^{2} \beta_{27} & \cdots & \left(\left(\alpha_{27}\right)^{2}\left(\beta_{27}\right)^{2}\left(\gamma_{27}\right)^{2}\right)
\end{array}\right]\left[\begin{array}{c}
A_{000} \\
A_{001} \\
\vdots \\
\vdots \\
A_{222}
\end{array}\right]} \\
& =\left[\begin{array}{c}
\left(v_{x y}\right)_{1} \\
\left(v_{x y}\right)_{2} \\
\vdots \\
\vdots \\
\left(v_{x y}\right)_{27}
\end{array}\right],
\end{aligned}
$$

where $\left(v_{x y}\right)_{i}$ is $v_{x y}$ of Model number $i$. Using the matrix form in (5), coefficients $A_{000}$ to $A_{222}$ can then be determined. Similarly, the coefficients of $v_{x z}$ function can be calculated from the following matrix form:

$$
\begin{gathered}
{\left[\begin{array}{cccc}
1 & \gamma_{1} \gamma_{1}^{2} \beta_{1} & \cdots & \left(\left(\alpha_{1}\right)^{2}\left(\beta_{1}\right)^{2}\left(\gamma_{1}\right)^{2}\right) \\
1 & \gamma_{2} \gamma_{2}{ }^{2} \beta_{2} & \cdots & \left(\left(\alpha_{2}\right)^{2}\left(\beta_{2}\right)^{2}\left(\gamma_{2}\right)^{2}\right) \\
\vdots & \vdots & \vdots & \vdots \\
\vdots & \vdots & \vdots & \vdots \\
1 & \gamma_{27} \gamma_{27}{ }^{2} \beta_{27} & \cdots & \left(\left(\alpha_{27}\right)^{2}\left(\beta_{27}\right)^{2}\left(\gamma_{27}\right)^{2}\right)
\end{array}\right]\left[\begin{array}{c}
B_{000} \\
B_{001} \\
\vdots \\
\vdots \\
B_{222}
\end{array}\right]} \\
=\left[\begin{array}{c}
\left(v_{x z}\right)_{1} \\
\left(v_{x z}\right)_{2} \\
\vdots \\
\vdots \\
\left(v_{x z}\right)_{27}
\end{array}\right],
\end{gathered}
$$


TABLE 1: Values of $\alpha, \beta$, and $\gamma$ used in analysed models.

\begin{tabular}{|c|c|c|c|}
\hline Model number & $\alpha($ radian $)$ & $\beta$ & $\gamma$ \\
\hline 1 & 0.8727 & 0.02 & 0.8 \\
\hline 2 & 0.8727 & 0.04 & 0.9 \\
\hline 3 & 0.8727 & 0.06 & 0.9 \\
\hline 4 & 0.9599 & 0.02 & 0.8 \\
\hline 5 & 0.9599 & 0.02 & 0.9 \\
\hline 6 & 0.9599 & 0.04 & 0.8 \\
\hline 7 & 0.9599 & 0.04 & 0.9 \\
\hline 8 & 0.9599 & 0.06 & 0.8 \\
\hline 9 & 0.9599 & 0.06 & 0.9 \\
\hline 10 & 1.0472 & 0.02 & 0.8 \\
\hline 11 & 1.0472 & 0.06 & 0.9 \\
\hline 12 & 1.1345 & 0.02 & 0.8 \\
\hline 13 & 1.1345 & 0.04 & 0.8 \\
\hline 14 & 1.1345 & 0.04 & 0.9 \\
\hline 15 & 1.1345 & 0.06 & 0.8 \\
\hline 16 & 1.309 & 0.02 & 0.8 \\
\hline 17 & 1.309 & 0.02 & 0.9 \\
\hline 18 & 1.309 & 0.04 & 0.8 \\
\hline 19 & 1.309 & 0.06 & 0.8 \\
\hline 20 & 1.309 & 0.06 & 0.9 \\
\hline 21 & 1.3963 & 0.02 & 0.8 \\
\hline 22 & 1.3963 & 0.02 & 0.9 \\
\hline 23 & 1.3963 & 0.04 & 0.9 \\
\hline 24 & 1.3963 & 0.06 & 0.8 \\
\hline 25 & 1.4835 & 0.02 & 0.8 \\
\hline 26 & 1.4835 & 0.02 & 0.9 \\
\hline 27 & 1.4835 & 0.04 & 0.8 \\
\hline
\end{tabular}

Obtaining $v_{y x}$ from finite element technique for all models also gives the coefficients of $v_{y x}$ function using the following matrix form:

$$
\begin{gathered}
{\left[\begin{array}{cccc}
1 & \gamma_{1} \gamma_{1}^{2} \beta_{1} & \cdots & \left(\left(\alpha_{1}\right)^{2}\left(\beta_{1}\right)^{2}\left(\gamma_{1}\right)^{2}\right) \\
1 & \gamma_{2} \gamma_{2}^{2} \beta_{2} & \cdots & \left(\left(\alpha_{2}\right)^{2}\left(\beta_{2}\right)^{2}\left(\gamma_{2}\right)^{2}\right) \\
\vdots & \vdots & \vdots & \vdots \\
\vdots & \vdots & \vdots & \vdots \\
1 & \gamma_{27} \gamma_{27}^{2} \beta_{27} & \cdots & \left(\left(\alpha_{27}\right)^{2}\left(\beta_{27}\right)^{2}\left(\gamma_{27}\right)^{2}\right)
\end{array}\right]\left[\begin{array}{c}
C_{000} \\
C_{001} \\
\vdots \\
\vdots \\
C_{222}
\end{array}\right]} \\
\quad=\left[\begin{array}{c}
\left(\nu_{y x}\right)_{1} \\
\left(v_{y x}\right)_{2} \\
\vdots \\
\vdots \\
\left(v_{y x}\right)_{27}
\end{array}\right],
\end{gathered}
$$

where $\left(v_{x z}\right)_{i}$ refers to $v_{x z}$ for Model number $i$.
Also, the coefficients of $v_{y z}$ function can be determined from the following matrix form:

$$
\begin{gathered}
{\left[\begin{array}{cccc}
1 & \gamma_{1} \gamma_{1}^{2} \beta_{1} & \cdots & \left(\left(\alpha_{1}\right)^{2}\left(\beta_{1}\right)^{2}\left(\gamma_{1}\right)^{2}\right) \\
1 & \gamma_{2} \gamma_{2}^{2} \beta_{2} & \cdots & \left(\left(\alpha_{2}\right)^{2}\left(\beta_{2}\right)^{2}\left(\gamma_{2}\right)^{2}\right) \\
\vdots & \vdots & \vdots & \vdots \\
\vdots & \vdots & \vdots & \vdots \\
1 & \gamma_{27} \gamma_{27}^{2} \beta_{27} & \cdots & \left(\left(\alpha_{27}\right)^{2}\left(\beta_{27}\right)^{2}\left(\gamma_{27}\right)^{2}\right)
\end{array}\right]\left[\begin{array}{c}
D_{000} \\
D_{001} \\
\vdots \\
\vdots \\
D_{222}
\end{array}\right]} \\
\quad=\left[\begin{array}{c}
\left(\nu_{y z}\right)_{1} \\
\left(v_{y z}\right)_{2} \\
\vdots \\
\vdots \\
\left(v_{y z}\right)_{27}
\end{array}\right] .
\end{gathered}
$$

Elastic modulus of all models along $x$ direction gives the coefficients of $\left(E_{1} / E\right)$ function $\left(E_{000}\right.$ to $\left.E_{222}\right)$ from the following equation:

$$
\begin{gathered}
{\left[\begin{array}{cccc}
1 & \gamma_{1} \gamma_{1}^{2} \beta_{1} & \cdots & \left(\left(\alpha_{1}\right)^{2}\left(\beta_{1}\right)^{2}\left(\gamma_{1}\right)^{2}\right) \\
1 & \gamma_{2} \gamma_{2}^{2} \beta_{2} & \cdots & \left(\left(\alpha_{2}\right)^{2}\left(\beta_{2}\right)^{2}\left(\gamma_{2}\right)^{2}\right) \\
\vdots & \vdots & \vdots & \vdots \\
\vdots & \vdots & \vdots & \vdots \\
1 & \gamma_{27} \gamma_{27}^{2} \beta_{27} & \cdots & \left(\left(\alpha_{27}\right)^{2}\left(\beta_{27}\right)^{2}\left(\gamma_{27}\right)^{2}\right)
\end{array}\right]\left[\begin{array}{c}
E_{000} \\
E_{001} \\
\vdots \\
\vdots \\
E_{222}
\end{array}\right]} \\
=\left[\begin{array}{c}
\left(\frac{E_{1}}{E}\right)_{1} \\
\left(\frac{E_{1}}{E}\right)_{2} \\
\vdots \\
\vdots \\
\left(\frac{E_{1}}{E}\right)_{27}
\end{array}\right] .
\end{gathered}
$$

Similarly, the following matrix form can be written to calculate coefficients of $E_{2} / E$ function $\left(F_{000}\right.$ to $\left.F_{222}\right)$ :

$$
\begin{gathered}
{\left[\begin{array}{cccc}
1 & \gamma_{1} \gamma_{1}^{2} \beta_{1} & \cdots & \left(\left(\alpha_{1}\right)^{2}\left(\beta_{1}\right)^{2}\left(\gamma_{1}\right)^{2}\right) \\
1 & \gamma_{2} \gamma_{2}^{2} \beta_{2} & \cdots & \left(\left(\alpha_{2}\right)^{2}\left(\beta_{2}\right)^{2}\left(\gamma_{2}\right)^{2}\right) \\
\vdots & \vdots & \vdots & \vdots \\
\vdots & \vdots & \vdots & \vdots \\
1 & \gamma_{27} \gamma_{27}^{2} \beta_{27} & \cdots & \left(\left(\alpha_{27}\right)^{2}\left(\beta_{27}\right)^{2}\left(\gamma_{27}\right)^{2}\right)
\end{array}\right]\left[\begin{array}{c}
F_{000} \\
F_{001} \\
\vdots \\
\vdots \\
F_{222}
\end{array}\right]} \\
=\left[\begin{array}{c}
\left(\frac{E_{2}}{E}\right)_{1} \\
\left(\frac{E_{2}}{E}\right)_{2} \\
\vdots \\
\vdots \\
\left(\frac{E_{2}}{E}\right)_{27}
\end{array}\right] .
\end{gathered}
$$




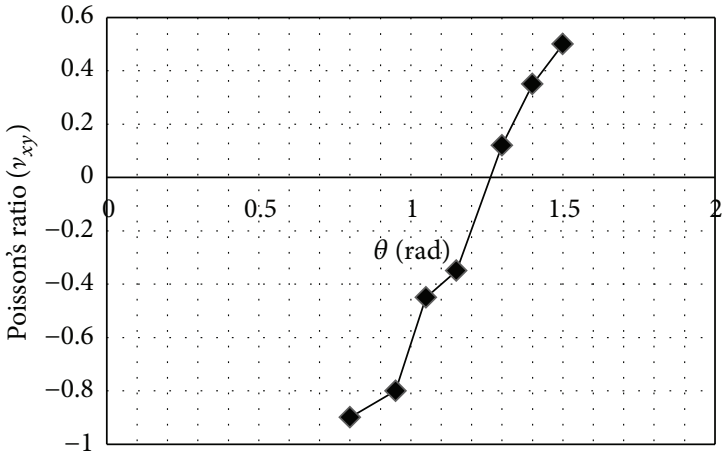

(a)

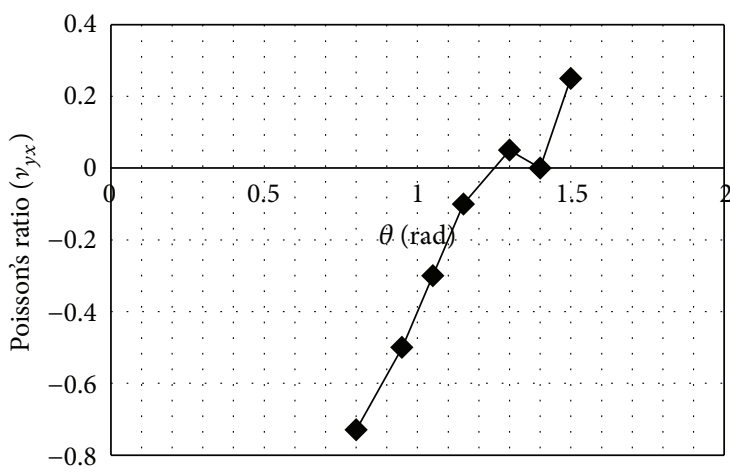

(c)

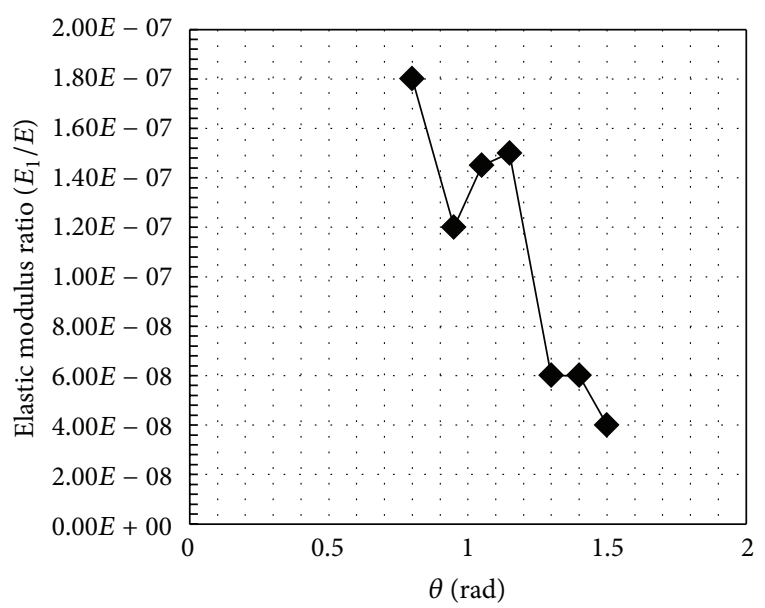

(e)

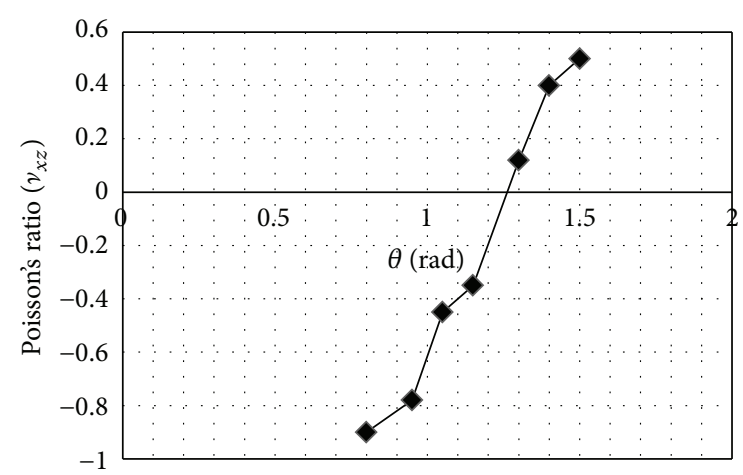

(b)

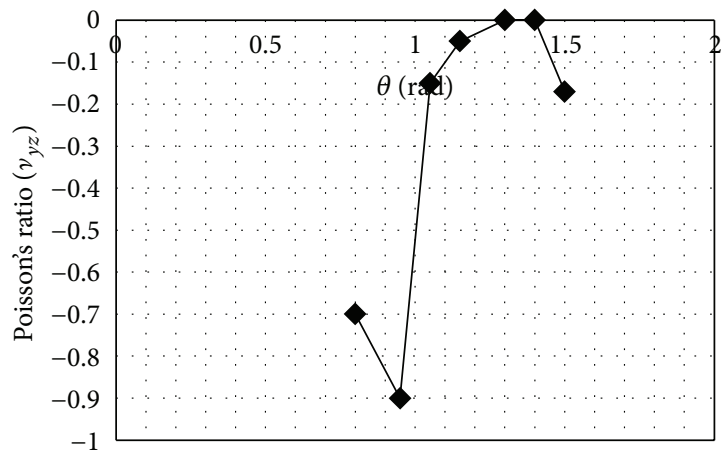

(d)

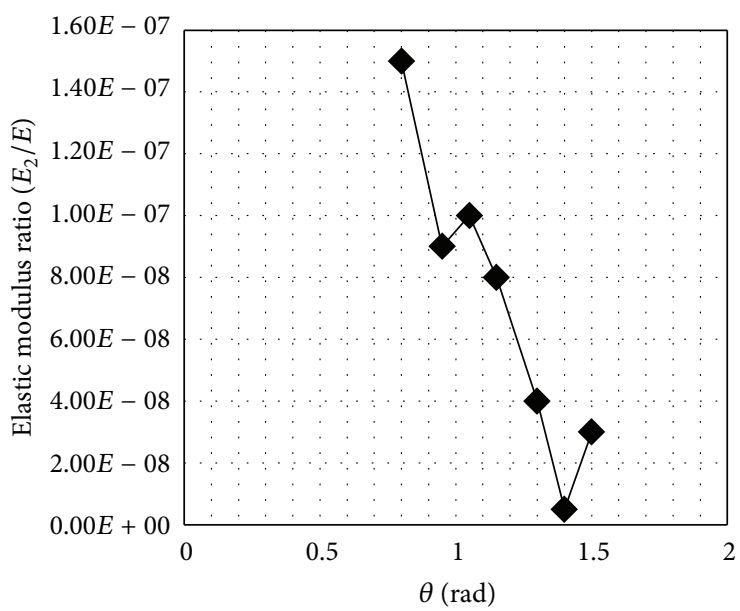

(f)

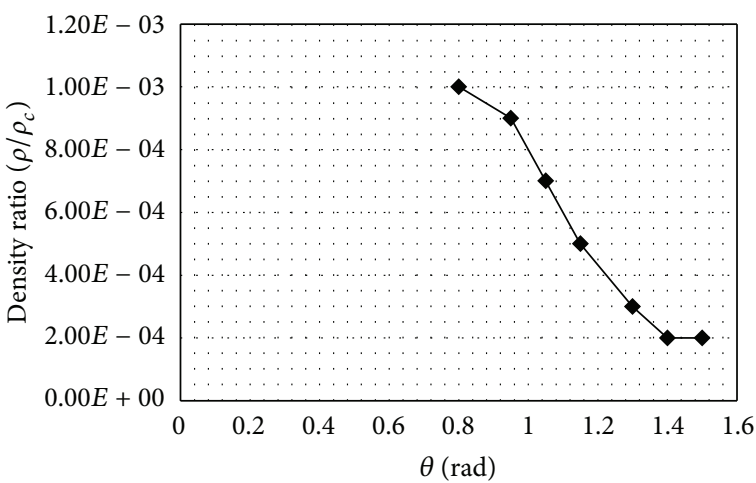

(g)

FIgURE 4: Mechanical properties versus $\theta$ in the case of fixing $\beta$ and $\gamma$ at 0.8 and 0.02 , respectively. 


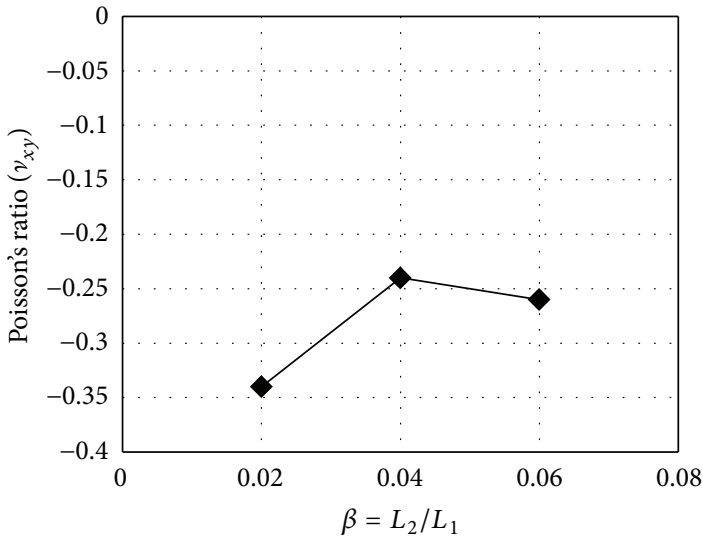

(a)

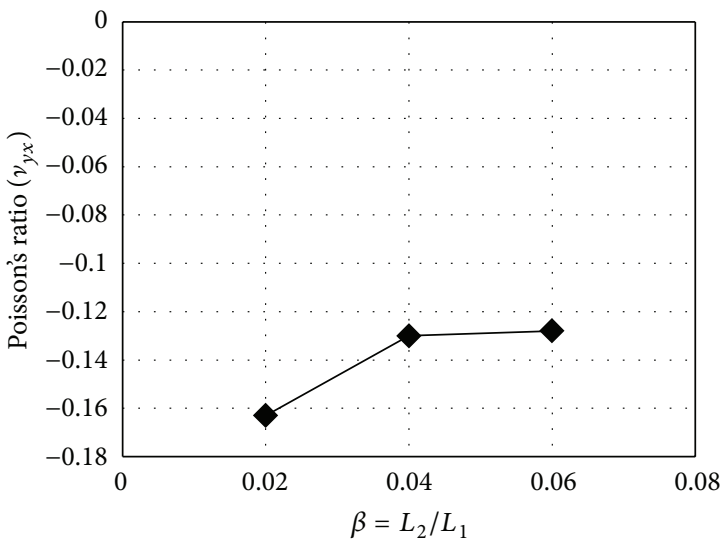

(c)

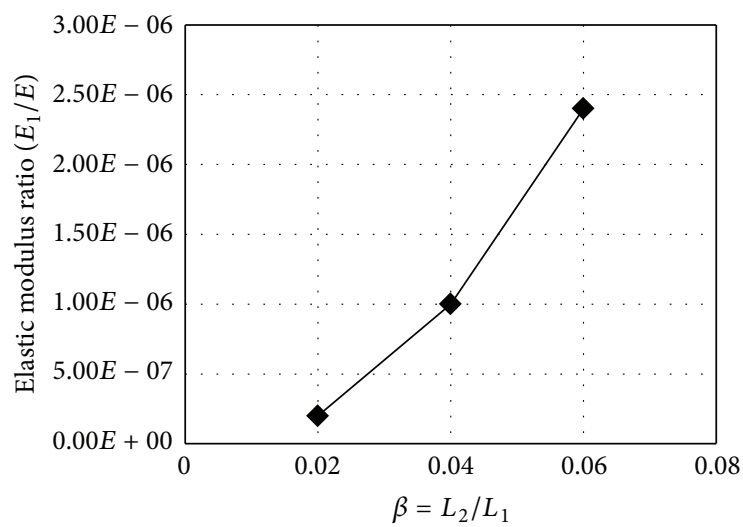

(e)

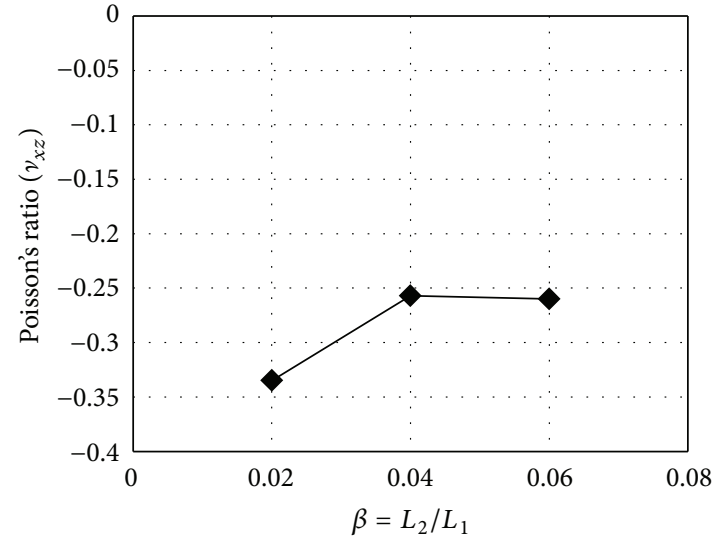

(b)

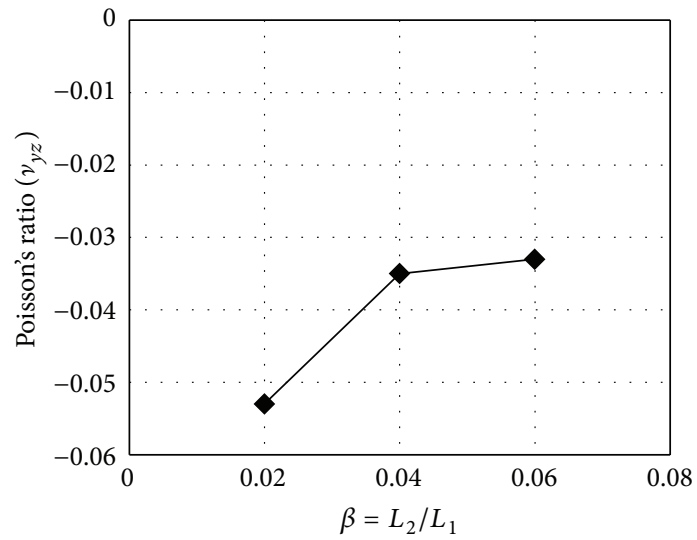

(d)

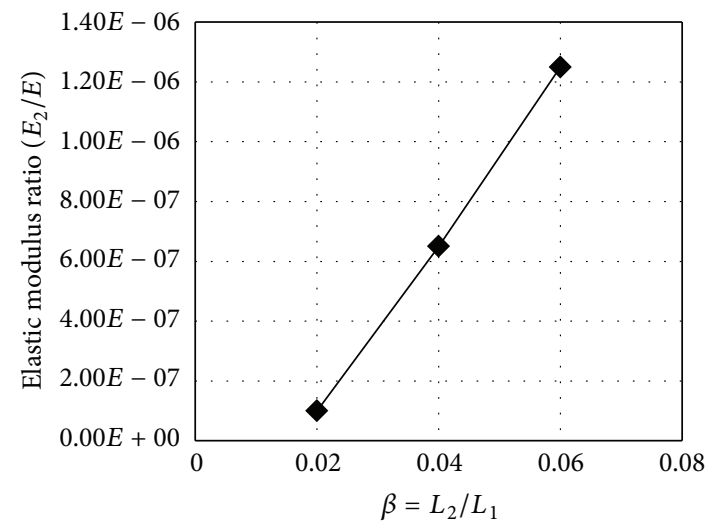

(f)

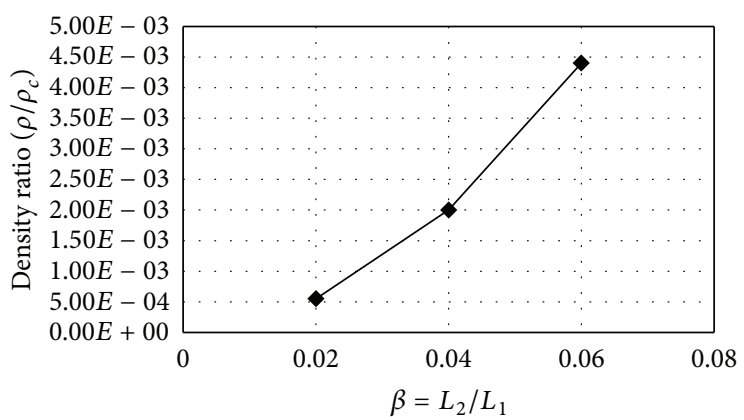

(g)

Figure 5: Mechanical properties versus $\beta$ in the case of fixing $\theta$ and $\gamma$ at $60^{\circ}$ and 0.02 , respectively. 
TABLE 2: Finite element results of Poisson's ratios, elastic modulus, and density ratio of models.

\begin{tabular}{|c|c|c|c|c|c|c|c|}
\hline Model & $v_{x y}$ & $v_{x z}$ & $v_{y x}$ & $v_{y z}$ & $E_{1} / E$ & $E_{2} / E$ & $\rho / \rho_{c}$ \\
\hline 1 & -0.885 & 0.905 & -0.7725 & -0.7215 & $18 e-8$ & $1.8 e-7$ & 0.001 \\
\hline 2 & -0.795 & 1.845 & -0.68 & -0.61 & $3.25 e-6$ & $2.63 e-6$ & 0.004 \\
\hline 3 & -0.945 & 0.745 & -0.7 & 0.1725 & $7.31 e-6$ & $5.89 e-6$ & 0.009 \\
\hline 4 & -0.76 & 0.785 & -0.5 & -0.385 & $1.2 e-7$ & $9 e-2$ & 0.0009 \\
\hline 5 & -0.75 & -0.74 & -0.5 & -0.4 & $2.6 e-7$ & $1.77 e-7$ & 0.0007 \\
\hline 6 & -0.705 & 0.69 & -0.478 & -0.325 & $2.32 e-6$ & $1.54 e-6$ & 0.0035 \\
\hline 7 & -0.69 & -0.705 & -0.485 & -0.315 & $2.18 e-6$ & $1.43 e-6$ & 0.0028 \\
\hline 8 & -0.7 & -0.715 & -0.47 & -0.36 & $4.36 e-6$ & $2.86 e-6$ & 0.0082 \\
\hline 9 & -0.69 & -0.705 & -0.545 & -0.32 & $4.83 e-6$ & $3.24 e-6$ & 0.0064 \\
\hline 10 & -0.461 & -0.46 & -0.3055 & -0.1465 & $1.4 e-7$ & $9.9 e-8$ & 0.0007 \\
\hline 11 & -0.485 & -0.5 & -0.294 & -0.1355 & $2.88 e-6$ & $1.61 e-6$ & 0.0047 \\
\hline 12 & -0.3415 & -0.335 & -0.163 & -0.0535 & $1.5 e-7$ & $7.4 e-8$ & 0.0005 \\
\hline 13 & -0.244 & -0.255 & -0.13 & -0.035 & $1.04 e-6$ & $5.68 e-7$ & 0.002 \\
\hline 14 & -0.269 & -0.2715 & -0.1365 & -0.0387 & $1.23 e-6$ & $6.11 e-7$ & 0.0016 \\
\hline 15 & -0.2575 & -0.255 & -0.1285 & -0.0333 & $2.36 e-6$ & $1.21 e-6$ & 0.0044 \\
\hline 16 & 0.095 & 0.092 & 0.062 & -0.01 & $6 e-7$ & $3.8 e-8$ & $3.8 e-8$ \\
\hline 17 & 0.1185 & 0.1165 & 0.065 & -0.0093 & $7 e-7$ & $4.5 e-8$ & 0.0003 \\
\hline 18 & 0.1695 & 0.1675 & 0.085 & -0.0083 & $6.3 e-7$ & $3.33 e-7$ & 0.0012 \\
\hline 19 & 0.1665 & 0.1695 & 0.09 & -0.1015 & $1.7 e-5$ & $7.41 e-7$ & 0.0027 \\
\hline 20 & 0.167 & 0.1655 & 0.09 & -0.015 & $1.33 e-6$ & $6.84 e-7$ & 0.0023 \\
\hline 21 & 0.2735 & 0.306 & 0.0034 & 0 & $6 e-8$ & $6 e-9$ & 0.0002 \\
\hline 22 & 0.2735 & 0.306 & 0.0034 & -0.04 & $2.5 e-7$ & $2.3 e-8$ & 0.001 \\
\hline 23 & 0.377 & 0.3665 & 0.178 & -0.06 & $5 e-8$ & $2.56 e-7$ & 0.0009 \\
\hline 24 & 0.335 & 0.385 & 0.1915 & -0.05 & $1.17 e-6$ & $6.2 e-7$ & 0.0022 \\
\hline 25 & 0.5 & 0.5 & 0.24 & 0.1535 & $4 e-8$ & $2.3 e-8$ & 0.0002 \\
\hline 26 & 0.3665 & 0.435 & 0.26 & -0.1165 & $5 e-8$ & 0 & 0.0002 \\
\hline 27 & 0.485 & 0.487 & 0.2735 & -0.131 & $4.3 e-7$ & 0 & 0.0008 \\
\hline
\end{tabular}

Density ratio coefficients can also be calculated from the following matrix form:

$$
\begin{gathered}
{\left[\begin{array}{cccc}
1 & \gamma_{1} \gamma_{1}^{2} \beta_{1} & \cdots & \left(\left(\alpha_{1}\right)^{2}\left(\beta_{1}\right)^{2}\left(\gamma_{1}\right)^{2}\right) \\
1 & \gamma_{2} \gamma_{2}^{2} \beta_{2} & \cdots & \left(\left(\alpha_{2}\right)^{2}\left(\beta_{2}\right)^{2}\left(\gamma_{2}\right)^{2}\right) \\
\vdots & \vdots & \vdots & \vdots \\
\vdots & \vdots & \vdots & \vdots \\
1 & \gamma_{27} \gamma_{27}^{2} \beta_{27} & \cdots & \left(\left(\alpha_{27}\right)^{2}\left(\beta_{27}\right)^{2}\left(\gamma_{27}\right)^{2}\right)
\end{array}\right]\left[\begin{array}{c}
G_{000} \\
G_{001} \\
\vdots \\
\vdots \\
G_{222}
\end{array}\right]} \\
=\left[\begin{array}{c}
\left(\frac{\rho}{\rho_{c}}\right)_{1} \\
\left(\frac{\rho}{\rho_{c}}\right)_{2} \\
\vdots \\
\vdots \\
\left(\frac{\rho}{\rho_{c}}\right)_{27}
\end{array}\right]
\end{gathered}
$$

where $\rho_{i}$ is density of Model number $i$ and $\rho_{c}$ is the density of the base material.
As illustrated, Poisson's ratio and elastic modulus of all defined models have been determined by using finite element approach. In terms of density ratio of the models, the formula of density for auxetic material was used to calculate density ratio. In particular, dependency of material density on geometrical parameters is presented as follows:

$$
\frac{\rho}{\rho_{c}}=\frac{M / V}{M / V_{c}}=\frac{a^{2}\left[6 L_{2}+16 L_{1}\right]}{\left[2 L_{2}+2 L_{1} \sin \theta-2 L_{1} \cos \theta\right]^{3}} .
$$

Using dimensionless geometrical parameters, (12) can be simplified as follows:

$$
\frac{\rho}{\rho_{c}}=\frac{\beta^{2}[3 \gamma+8]}{4[\gamma+\sin \alpha-\cos \alpha]^{3}} .
$$

After calculating the mechanical properties for all models, the coefficients $A_{i j k}$ to $G_{i j k}$ were then determined using (5) to (11). 
TABLE 3: Coefficients of Poisson's ratios, elastic modulus, and density ratio functions obtained from (5) to (11).

\begin{tabular}{|c|c|c|c|c|c|c|c|c|c|}
\hline$i$ & $j$ & $k$ & $A_{i j k}$ & $B_{i j k}$ & $C_{i j k}$ & $D_{i j k}$ & $E_{i j k}$ & $F_{i j k}$ & $G_{i j k}$ \\
\hline 0 & 0 & 0 & -7.2168 & -12.856 & -1.3786 & -1.6593 & $-0.35 e-4$ & $-0.49 e-6$ & -0.0043 \\
\hline 0 & 0 & 1 & 1.1382 & -2.6137 & -0.6491 & -0.8715 & $-0.06 e-4$ & $0.12 e-6$ & 0.0023 \\
\hline 0 & 0 & 2 & 5.0877 & -2.3516 & -2.9302 & 0.4541 & $-0.08 e-4$ & $-0.03 e-6$ & -0.0003 \\
\hline 0 & 1 & 0 & -1.54 & -3.2677 & -1.6192 & -1.8352 & $-0.06 e-4$ & $0.16 e-6$ & 0.0003 \\
\hline 0 & 1 & 1 & 1.38 & 7.126 & 0.7405 & 2.8019 & $0.11 e-4$ & $-0.23 e-6$ & -0.0028 \\
\hline 0 & 1 & 2 & -2.178 & -0.2933 & 3.1445 & 0.0164 & $0.15 e-4$ & $0.09 e-6$ & 0.0016 \\
\hline 0 & 2 & 0 & 7.973 & 3.4427 & -2.4428 & -0.105 & $0.39 e-4$ & $0.67 e-6$ & 0.0031 \\
\hline 0 & 2 & 1 & -5.746 & 0.2005 & 0.1502 & -2.429 & $-0.05 e-4$ & $0.29 e-6$ & 0.0012 \\
\hline 0 & 2 & 2 & 0.6389 & 10.103 & 4.5258 & 3.2359 & $-0.04 e-4$ & $-0.49 e-6$ & -0.0005 \\
\hline 1 & 0 & 0 & 1.0689 & 6.546 & 2.3045 & 1.7954 & 0.0065 & $-0.13 e-6$ & -0.0005 \\
\hline 1 & 0 & 1 & 0.5116 & -6.793 & -2.7854 & -1.8819 & $0.16 e-4$ & $0.28 e-6$ & 0.0016 \\
\hline 1 & 0 & 2 & 2.3861 & -7.7101 & 2.3285 & 1.4796 & $0.14 e-4$ & $0.03 e-6$ & -0.0011 \\
\hline 1 & 1 & 0 & -1.9467 & -1.0019 & 4.3855 & 0.3243 & $-0.06 e-4$ & $-0.13 e-6$ & 0.0005 \\
\hline 1 & 1 & 1 & 0.2927 & 5.9708 & 1.3435 & 1.8795 & $0.18 e-4$ & $-0.01 e-6$ & 0.0005 \\
\hline 1 & 1 & 2 & -2.3464 & -7.1761 & -2.4561 & -2.3187 & $-0.16 e-4$ & $0.13 e-6$ & 0.0007 \\
\hline 1 & 2 & 0 & 1.0728 & 1.3113 & -2.6061 & 1.53 & $0.22 e-4$ & $-0.09 e-6$ & -0.0021 \\
\hline 1 & 2 & 1 & 2.4819 & -5.5095 & 0.1735 & 1.9218 & $-0.02 e-4$ & $-0.28 e-6$ & -0.0033 \\
\hline 1 & 2 & 2 & -3.5178 & -1.0343 & -2.7304 & -4.7579 & $-0.46 e-4$ & $0.27 e-6$ & 0.0036 \\
\hline 2 & 0 & 0 & -1.8739 & 2.2626 & 3.3666 & 1.0313 & $00.1 e-4$ & $-0.16 e-6$ & -0.0009 \\
\hline 2 & 0 & 1 & 5.3769 & 8.8403 & -0.3328 & 1.6796 & $-0.01 e-4$ & $-0.03 e-6$ & 0.0014 \\
\hline 2 & 0 & 2 & -5.8394 & -0.6419 & -0.3505 & -2.1122 & $0.12 e-4$ & $0.52 e-6$ & 0.0022 \\
\hline 2 & 1 & 0 & 4.0336 & 1.1187 & -1.4741 & -0.8057 & $-0.07 e-4$ & $0.16 e-6$ & 0.0018 \\
\hline 2 & 1 & 1 & -2.0998 & 1.8141 & -2.9387 & -3.4479 & $-0.34 e-4$ & $0.18 e-6$ & 0.0021 \\
\hline 2 & 1 & 2 & 3.4834 & -4.8668 & -0.8514 & 3.2488 & $0.23 e-4$ & $-0.35 e-6$ & -0.0047 \\
\hline 2 & 2 & 0 & -2.0249 & 2.0253 & -0.6306 & -0.5081 & $-0.17 e-4$ & $0.03 e-6$ & 0.0022 \\
\hline 2 & 2 & 1 & -4.3087 & -9.6861 & 4.9212 & 0.1893 & $0.07 e-4$ & $-0.27 e-6$ & -0.0027 \\
\hline 2 & 2 & 2 & 3.2529 & -0.8663 & -1.7096 & 0.7249 & $0.07 e-4$ & $-0.08 e-6$ & -0.0014 \\
\hline
\end{tabular}

\section{Numerical Analysis of Auxetic Star Honeycomb Structure}

This present study has focused on Poisson's ratio, Young's modulus, and the density of a $3 \mathrm{D}$ element of auxetic material made of star honeycomb structure using numerical method in conjunction with analytical approach. Details of the results obtained for the models consisting of cells in 27 cases are also presented in Table 1 . Some of the numerical results are in details shown in Table 2 and Figures 2, 3, 4, and 5.

The primary outcomes of the finite element analyses could be remarked as follows.

(i) From $\theta=45^{\circ}$ to $70^{\circ}$, the structure exhibits auxetic behaviour in all directions.

(ii) From $\theta=70^{\circ}$ to $90^{\circ}, v_{y z}$ is negative and other Poisson's ratios are positive.

(iii) From $\theta=45^{\circ}$ to $70^{\circ}$, the auxeticity decreases as the angle $\theta$ increases.

(iv) In terms of elastic modulus and density, $E$ and $\rho$ decrease with the increasing of $\theta$.

(v) Dimensionless geometrical parameters used in this research are $\alpha=\theta, \beta=L_{2} / L_{1}$, and $\gamma=a / L_{1}$. Taking into consideration the values of these parameters used for formulation, using the obtained formulas for designing star-shaped structures in which domain of dimensionless geometrical parameters is $0.8202 \leq$ $\alpha \leq 1.5184,0.8 \leq \beta \leq 0.9$, and $0.02 \leq$ $\gamma \leq 0.06$ leads to reasonable results. However, these formulas can only be used to estimate the mechanical properties of star honeycomb structures with dimensionless geometric parameters close to the values of the parameters used in the formulation.

(vi) As illustrated, the aim of this study is to introduce a method for formulating beam like star honeycomb structures. Although the result of that is reasonable for limited domain of geometrical parameters, it offers a method for formulating mechanical properties for any desired domain in which the auxetic material is about to be designed and fabricated.

(vii) Although the shape of the defined structure seems to exhibit auxetic behaviour in all directions, it has interestingly found that it does not exhibit auxetic behaviour in some cases depending on the values of geometrical parameters. For instance, from $\theta=$ $70^{\circ}$ onward, the structure does not exhibit auxetic behaviour. 
For further details, the result of formulation used to obtain mathematical formula of mechanical properties is shown in Table 3.

\section{Conclusion}

A symmetrical configuration for the star honeycomb cellular structure of an auxetic material has been proposed and subsequently its analytical principle has also been formulated. The topology allows more degrees of freedom to the designer in controlling the mechanical properties of auxetic material by varying the wall lengths, cell wall thickness, and angle. Manipulation of stiffness properties including Poisson's ratio and fracture toughness can be performed by modification of the cellular structure's structural parameters. The overall mechanical properties can be controlled by modification of the base wall angle of the configurations, with the consequent changes, in particular, of the in-plane Poisson's ratio. This research provides a guideline for applications to $3 \mathrm{D}$ star honeycomb auxetic structures and also provides a basis for experimental additive manufacturing processes such as electron beam melting or selective laser sintering.

\section{Appendix}

$f_{1}$ : Function of $v_{x y}$ versus geometrical parameters.

$f_{2}$ : Function of $v_{x z}$ versus geometrical parameters.

$f_{3}$ : Function of $v_{y x}$ versus geometrical parameters.

$f_{4}$ : Function of $v_{y z}$ versus geometrical parameters.

$f_{5}$ : Function of $E_{1}$ versus geometrical parameters.

$f_{6}$ : Function of $E_{2}$ versus geometrical parameters.

$f_{7}$ : Function of $\rho$ versus geometrical parameters.

\section{Conflict of Interests}

The authors declare that there is no conflict of interests regarding the publication of this paper.

\section{Acknowledgment}

Funding by the Ministry of Education (MOE), Government of Malaysia through Universiti Teknologi Malaysia, research Grants R.J130000.7824.4L105 and R.J130000.7824.4F248 are sincerely acknowledged.

\section{References}

[1] J. Lee, J. B. Choi, and K. Choi, "Application of homogenization FEM analysis to regular and re-entrant honeycomb structures," Journal of Materials Science, vol. 31, no. 15, pp. 4105-4110, 1996.

[2] N. Gaspar, X. J. Ren, C. W. Smith, J. N. Grima, and K. E. Evans, "Novel honeycombs with auxetic behaviour," Acta Materialia, vol. 53, no. 8, pp. 2439-2445, 2005.

[3] L. J. Gibson, M. F. Ashby, G. S. Schajer, and C. I. Robertson, “The mechanics of two dimensional cellular materials," Proceedings of The Royal Society of London, Series A: Mathematical and Physical Sciences, vol. 382, no. 1782, pp. 25-42, 1982.
[4] P. S. Theocaris and G. E. Stavroulakis, "The homogenization method for the study of variation of Poisson's ratio in fiber composites," Archive of Applied Mechanics, vol. 68, no. 3-4, pp. 281-295, 1998.

[5] T.-C. Lim, "Constitutive relationship of a material with unconventional Poisson's ratio," Journal of Materials Science Letters, vol. 22, no. 24, pp. 1783-1786, 2003.

[6] Y. Li, “The anisotropic behavior of Poisson's ratio, Young's modulus, and shear modulus in hexagonal materials," Physica Status Solidi A, vol. 38, no. 1, pp. 171-175, 1976.

[7] J. N. Grima, R. Gatt, A. Alderson, and K. E. Evans, "On the potential of connected stars as auxetic systems," Molecular Simulation, vol. 31, no. 13, pp. 925-935, 2005.

[8] J. N. Grima, R. Gatt, and P.-S. Farrugia, "On the properties of auxetic meta-tetrachiral structures," Physica Status Solidi (B) Basic Research, vol. 245, no. 3, pp. 511-520, 2008.

[9] D. Prall and R. S. Lakes, "Properties of a chiral honeycomb with a poisson's ratio of -1," International Journal of Mechanical Sciences, vol. 39, no. 3, pp. 305-314, 1997.

[10] A. Spadoni, M. Ruzzene, and F. Scarpa, "Global and local linear buckling behavior of a chiral cellular structure," Physica Status Solidi (b), vol. 242, no. 3, pp. 695-709, 2005.

[11] D. Bornengo, F. Scarpa, and C. Remillat, "Evaluation of hexagonal chiral structure for morphine airfoil concept," Proceedings of the Institution of Mechanical Engineers, Part G: Journal of Aerospace Engineering, vol. 219, no. 3, pp. 185-192, 2005.

[12] Y. Ishibashi and M. Iwata, "A microscopic model of a negative Poisson's ratio in some crystals," Journal of the Physical Society of Japan, vol. 69, no. 8, pp. 2702-2703, 2000.

[13] A. A. Vasiliev, S. V. Dimitriev, Y. Ishibashi, and T. Shinegari, "Elastic properties of a two-dimensional model of crystals containing particles with rotational degrees of freedom," Physical Review B, vol. 65, Article ID 094101, 2002.

[14] J. N. Grima, R. Gatt, N. Ravirala, A. Alderson, and K. E. Evans, "Negative Poisson's ratios in cellular foam materials," Materials Science and Engineering A, vol. 423, no. 1-2, pp. 214-218, 2006.

[15] J. N. Grima, R. Gatt, A. Alderson, and K. E. Evans, "On the auxetic properties of "rotating rectangles" with different connectivity," Journal of the Physical Society of Japan, vol. 74, no. 10, pp. 2866-2867, 2005.

[16] G. W. Milton, "Composite materials with Poisson's ratios close to-1," Journal of the Mechanics and Physics of Solids, vol. 40, pp. 1105-1137, 1992.

[17] P. J. Hine, R. A. Duckett, and I. M. Ward, "Negative Poisson's ratios in angle-ply laminates," Journal of Materials Science Letters, vol. 16, no. 7, pp. 541-544, 1997.

[18] K. W. Wojciechowski, "Constant thermodynamic tension Monte Carlo studies of elastic properties of a two-dimensional system of hard cyclic hexamers," Molecular Physics, vol. 61, no. 5, pp. 1247-1258, 1987.

[19] K. W. Wojciechowski, "Remarks on 'Poisson ratio beyond the limits of the elasticity theory," Journal of the Physical Society of Japan, vol. 72, no. 7, pp. 1819-1820, 2003.

[20] K. W. Wojciechowski, "Non-chiral, molecular model of negative Poisson ratio in two dimensions," Journal of Physics A: Mathematical and General, vol. 36, no. 47, pp. 11765-11778, 2003.

[21] K. V. Tretiakov and K. W. Wojciechowski, "Poisson's ratio of simple planar 'isotropic' solids in two dimensions," Physica Status Solidi B: Basic Research, vol. 244, no. 3, pp. 1038-1046, 2007. 
[22] B. D. Caddock and K. E. Evans, "Microporous materials with negative Poisson's ratios. I. Microstructure and mechanical properties," Journal of Physics D: Applied Physics, vol. 22, no. 12, pp. 1877-1882, 1989.

[23] A. Alderson and K. L. Alderson, "Auxetic materials," Proceedings of the Institution of Mechanical Engineers, Part G: Journal of Aerospace Engineering, vol. 221, no. 4, pp. 565-575, 2007.

[24] K. L. Alderson, A. Alderson, and K. E. Evans, "The interpretation of the strain-dependent Poisson's ratio in auxetic polyethylene," Journal of Strain Analysis for Engineering Design, vol. 32, no. 3, pp. 201-212, 1997.

[25] C. He, P. Liu, P. J. McMullan, and A. C. Griffin, "Toward molecular auxetics: main chain liquid crystalline polymers consisting of laterally attached para-quaterphenyls," Physica Status Solidi (B) Basic Research, vol. 242, no. 3, pp. 576-584, 2005.

[26] P. Aldred and S. C. Moratti, "Dynamic simulations of potentially auxetic liquid-crystalline polymers incorporating swivelling mesogens," Molecular Simulation, vol. 31, no. 13, pp. 883-887, 2005.

[27] Y. Liu and H. Hu, "A review on auxetic structures and polymeric materials," Scientific Research and Essays, vol. 5, no. 10, pp. 10521063, 2010.

[28] P. S. Theocaris, G. E. Stavroulakis, and P. D. Panagiotopoulos, "Negative Poisson's ratios in composites with star-shaped inclusions: a numerical homogenization approach," Archive of Applied Mechanics, vol. 67, no. 4, pp. 274-286, 1997. 

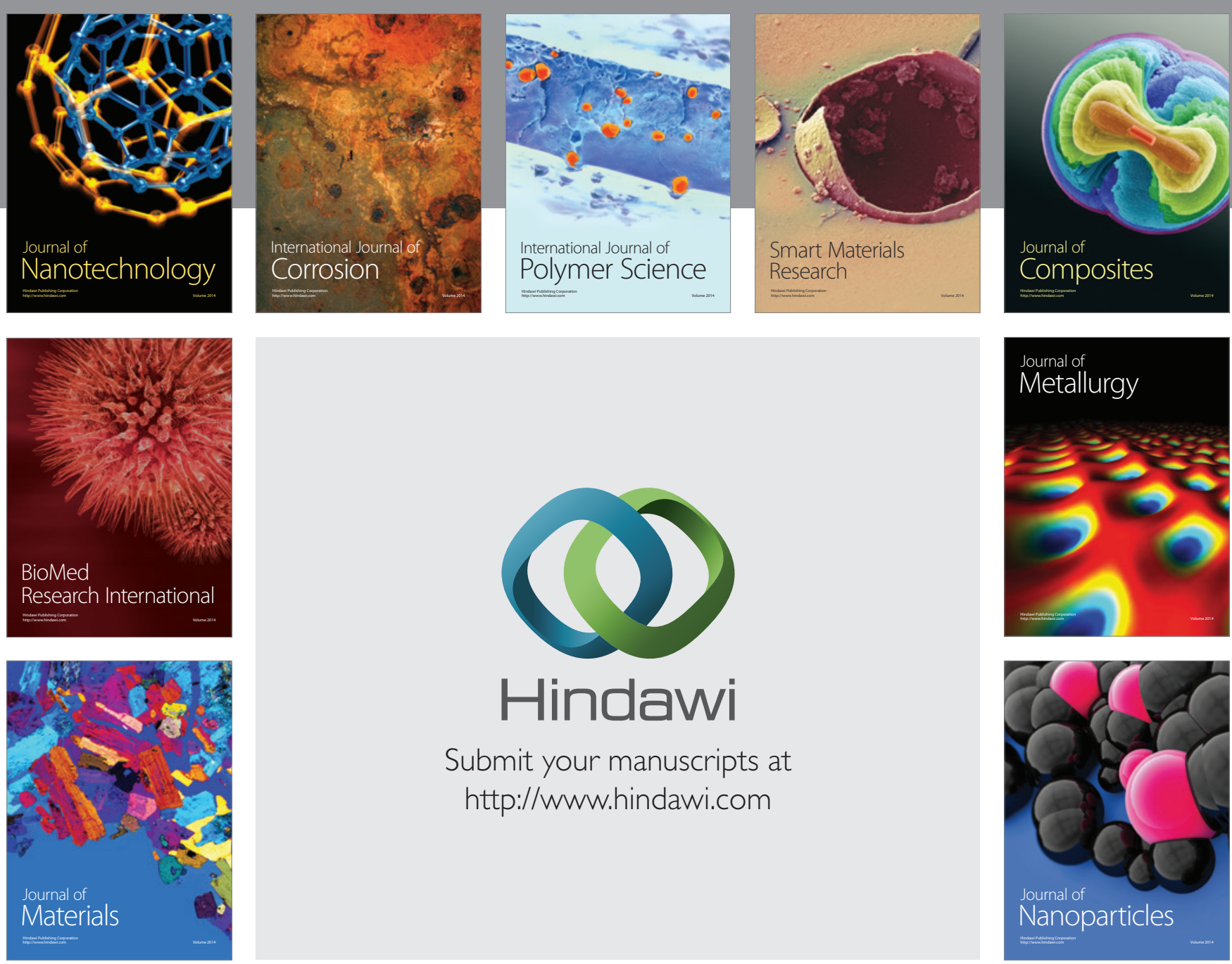

Submit your manuscripts at http://www.hindawi.com
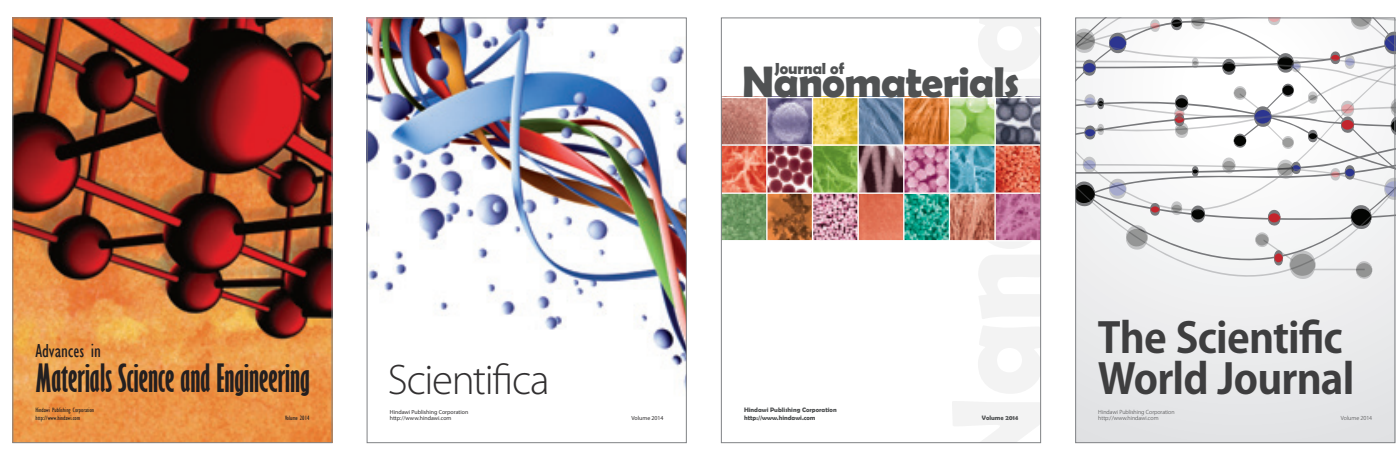

\section{The Scientific World Journal}
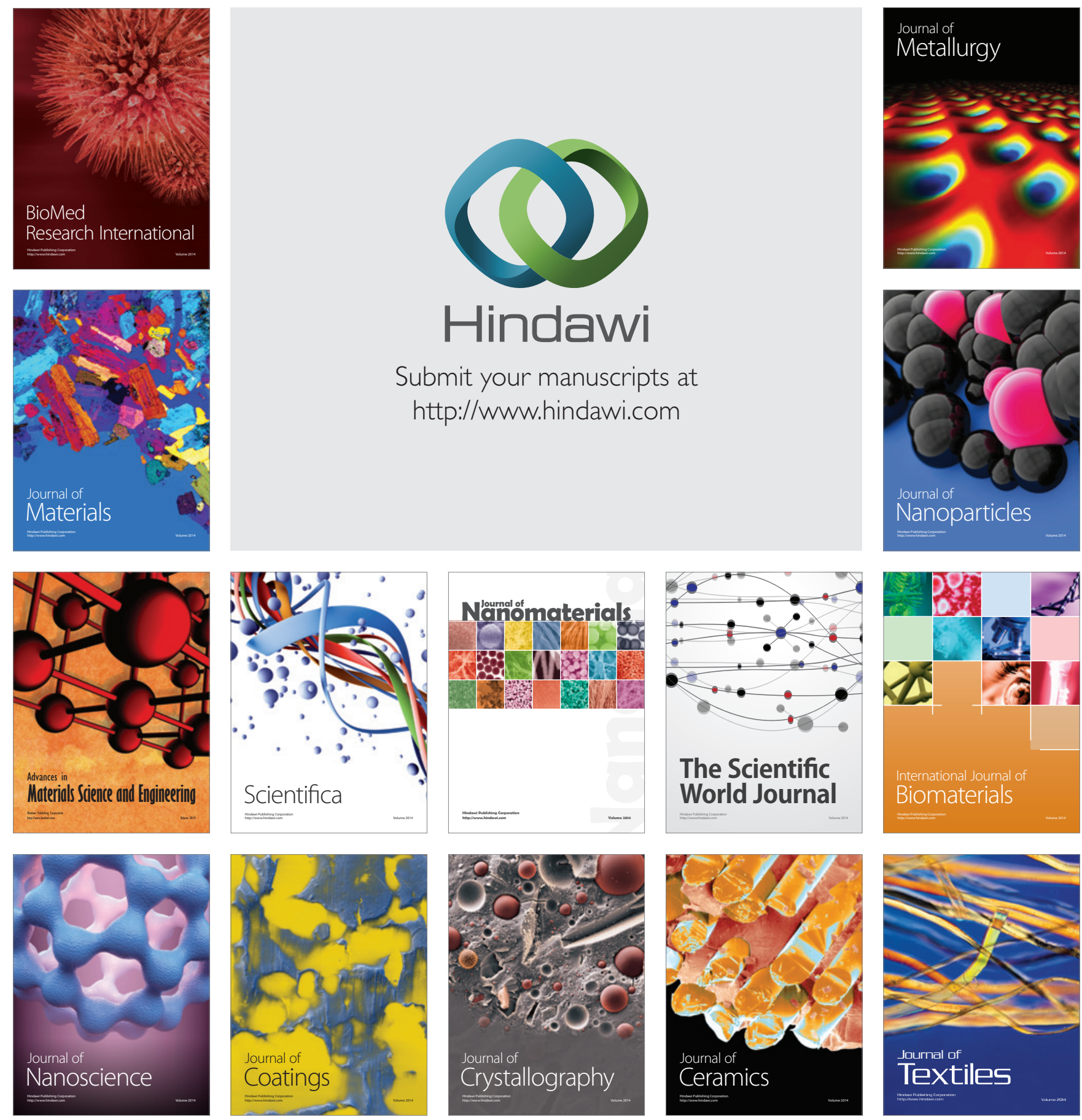